Sharif University of Technology
Scientia Iranica
Transactions E: Industrial Engineering
wCIENTIA

\title{
Step change point estimation of the first-order autoregressive autocorrelated simple linear profiles
}

\author{
R. Baradaran Kazemzadeh ${ }^{\mathrm{a}, *}$, A. Amiri ${ }^{\mathrm{b}}$ and H. Mirbeik ${ }^{\mathrm{a}}$ \\ a. Department of Industrial Engineering, Faculty of Engineering, Tarbiat Modares University, Tehran, Iran. \\ b. Department of Industrial Engineering, Faculty of Engineering, Shahed University, Tehran, Iran. \\ Received 21 November 2014; received in revised form 12 June 2015; accepted 26 December 2015
}

\author{
KEYWORDS \\ Simple linear profile; \\ Autocorrelation; \\ Step change point; \\ $\operatorname{AR}(1)$ \\ Phase II.
}

\begin{abstract}
In most researches in area of profile monitoring, it is assumed that observations are independent of each other, whereas this assumption is usually violated in practice; observations are autocorrelated. The control charts are the most important tools of the statistical process control which are used to monitor the processes over time. The control charts usually signal the out-of-control status of the process with a time delay. While knowing real-time of the change (change point), one can achieve great savings on time and expenses. In this paper, the estimation of the change point in simple linear profiles with $\mathrm{AR}(1)$ autocorrelation structure within each profile is considered. In the proposed method, by acquiring the joint probability density function of the autocorrelated observations, the maximum likelihood estimation method is applied to estimate the step change point. Here, we specifically focus on Phase II and compare the performance of the proposed estimator with the existing estimators in the literature through simulation studies. In addition, the application of the proposed estimator in comparison with the two estimators is illustrated through a real case. The results show the better performance of the proposed estimator.

(C) 2016 Sharif University of Technology. All rights reserved.
\end{abstract}

\section{Introduction}

Sometimes, quality of a process or product is described by a functional relationship between two or more quality characteristics referred to as profile [1]. The simple linear profile is one of the various types of profiles which is expressed as relationship between a response variable and an explanatory variable. This type of profile is more attractive to researchers due to their simplicity and applicability, especially in calibration applications. One of the assumptions which is considered in most of the researches in this area is the independency of observations within each profile. However, this assumption is violated in many real

*. Corresponding author. Tel./Fax: +98 2182883537 E-mail addresses: rkazem@modares.ac.ir (R. Baradaran Kazemzadeh); amiri@shahed.ac.ir (A. Amiri);

hr.mirbeik@att.net (H. Mirbeik) situations, especially when samples are taken in short intervals of time [2]. Numerous studies are done to monitor the autocorrelated profiles in both Phases I and II. Jensen et al. [3] applied the linear mixed models to monitor the autocorrelated linear profiles in Phase I control chart applications. Noorossana et al. [4] proposed three methods to monitor simple linear profiles with autocorrelation between successive profiles in Phase II. Soleimani et al. [1] considered the autocorrelated simple linear profiles and proposed a remedial measure to eliminate the effect of $\operatorname{AR}(1)$ autocorrelation between observations in each profile. Other researches on monitoring autocorrelated profiles include Kazemzadeh et al. [5], Noorossana et al. [6], Soleimani et al. [7], Narvand et al. [8], Koosha and Amiri [2], and Zhang et al. [9]. On the other hand, once the control chart signals the presence of a change in the process, the search for detecting the assignable causes is started. Usually, the alarm time does not 
coincide with the real time of change in the process. Finding the real time of change in the process simplifies the process of finding the root cause of control chart signal and decreases the risk of misdiagnosing the alarm by the control chart [10]. The real time of changes in the process is referred to as change point. The topic of the change point estimation has been discussed by many researchers such as Nedumaran et al. [11], Sullivan [12], Perry and Pignatiello [13,14], Ghazanfari et al. [15], Noorossana and Shadman [16], Torkamani et al. [17], and so on. For more information about different studies in the area of change point estimation after a signal by control charts refer to Amiri and Allahyari [10]. Estimation of the change point in the area of profile monitoring is also discussed by many researchers. Mahmoud et al. [18] proposed the change point approach to monitor the regression parameters in a simple linear profile in Phase I. Sharafi et al. [19] used Maximum Likelihood Estimator (MLE) to estimate the real time of a drift change in the parameters of a logistic regression profile. Zand et al. [20] developed two methods to estimate the real time of a step change in Phase I monitoring of the logistic profiles. Sharafi et al. [21] proposed the use of an MLE to estimate a step change in the parameters of Poisson regression profiles. Kazemzadeh et al. [22] developed the Maximum Likelihood Estimators (MLEs) for both step change and linear drift in the regression parameters of multivariate linear profiles.

There are also few researches in field of the change point estimation in the autocorrelated profiles. Keramatpour et al. [23] considered the polynomial profiles with autocorrelated structure of the first-order autoregressive. At first, they proposed a remedial measure to diminish the effect of autocorrelation in Phase II monitoring of autocorrelated polynomial profiles. Then, they suggested a control chart based on the Generalized Linear Test (GLT) for monitoring the coefficients of polynomial profiles, and an R-chart was used to monitor the error variance. Finally, they proposed an estimator of likelihood ratio to estimate the changes in the parameters of the autocorrelated polynomial profiles. Kazemzadeh et al. [24] applied two estimators, including MLE and clustering, to estimate the step change point in monitoring of AR(1) autocorrelated simple linear profiles. They used the transformation method to omit the autocorrelation effect of the observation within each profile. Finally, they evaluated and compared the performance of the two proposed estimators by simulation. In this paper, a maximum likelihood estimator based on the joint probability density function of the $\operatorname{AR}(1)$ observations in each profile is proposed to estimate the step change point. Also, the performance of the proposed estimator is compared with the two estimators discussed by Kazemzadeh et al. [24] through simulation studies. Fi- nally, a real case is presented to show the applicability of the proposed estimator. The structure of the paper is as follows.

In Section 2, formulation of the problem model is presented and the assumptions are expressed. The $T^{2}$ control chart for monitoring the first-order autoregressive autocorrelated simple linear profiles is explained in Section 3. In Section 4, the existing methods for estimating the change point in $\operatorname{AR}(1)$ autocorrelated simple linear profiles are discussed. In Section 5, the proposed estimator for the estimation of the change point is described. In Section 6, the performance of the proposed estimator is compared with the performance of the existing methods through simulation studies. A real case is discussed in Section 7. Our concluding remarks are presented in the final section.

\section{Problem formulation}

Similar to Soleimani et al. [1], we suppose that for $j$ th sample gathered over time, we have observations $\left(x_{i}, y_{i j}\right), i=1,2, \cdots, n$. When the process is under statistical control, then the relationship between the response and independent variables is as in Eqs. (1) and (2):

$$
\begin{aligned}
& y_{i j}=A_{0}+A_{1} x_{i}+\varepsilon_{i j}, \\
& \varepsilon_{i j}=\rho \varepsilon_{(i-1) j}+u_{i j},
\end{aligned}
$$

where $\varepsilon_{i j}$ s are the autocorrelated error terms with firstorder autoregressive structure, and $u_{i j} \mathrm{~s}$ are normal random variables with mean zero and variance $\sigma^{2}\left(u_{i j} \sim\right.$ $N I\left(0, \sigma^{2}\right)$. It is assumed that $x$-values are fixed and constant from profile to profile. Also, Phase II monitoring analysis is considered in this paper. In other words, it is assumed that $A_{0}, A_{1}$, and $\sigma$ values are known parameters. There is the first order autoregressive autocorrelation structure within the profile, and the autocorrelation between profiles does not exist.

Here, we use the transformation method of Soleimani et al. [1] to deal with the effects of autocorrelation. The observation in each profile can be stated as:

$$
y_{i j}=A_{0}+A_{1} x_{i}+\varepsilon_{i j} .
$$

Also, we have:

$$
y_{(i-1) j}=A_{0}+A_{1} x_{i-1}+\varepsilon_{(i-1) j} .
$$

By replacing Eqs. (2) and (4) in Eq. (3), we have:

$$
y_{i j}^{\prime}=\left(A_{0}+A_{1} x_{i}+\varepsilon_{i j}\right)-\rho\left(A_{0}+A_{1} x_{i-1}+\varepsilon_{(i-1) j}\right),
$$

where $y_{i j}^{\prime}=y_{i j}-\rho y_{(i-1) j}$. After simplifying Eq. (5), we have:

$$
y_{i j}^{\prime}=A_{0}(1-\rho)+A_{1}\left(x_{i}-\rho x_{i-1}\right)+\left(\varepsilon_{i j}-\rho \varepsilon_{(i-1) j}\right) .
$$


Therefore, considering the following relationship:

$$
u_{i j}=\varepsilon_{i j}-\rho \varepsilon_{(i-1) j},
$$

we have:

$$
\begin{aligned}
& y_{i j}^{\prime}=A_{0}^{\prime}+A_{1}^{\prime} x_{i}^{\prime}+u_{i j}, \\
& y_{i j}^{\prime}=A_{o}(1-\rho)+A_{1}\left(x_{i}-\rho x_{i-1}\right)+u_{i j},
\end{aligned}
$$

where $u_{i j}$ 's are independent random variables with mean zero and variance of $\sigma^{2}$ and $A_{0}^{\prime}=A_{0}(1-\rho), A_{1}^{\prime}=$ $A_{1}$. Thus, we obtain a simple linear profile model where the corresponding residuals are independent of each other. Hence, by using the transformed variables $x_{i}^{\prime}$, and $y_{i j}^{\prime}$, a simple linear profile model with independent residuals is obtained. Then, we can use the ordinary square (OLS) to estimate the regression parameters.

\section{Monitoring procedure: $T^{2}$ control chart}

To monitor the autocorrelated profiles, we apply the $T^{2}$ control chart by Kang and Albin [25]. For this purpose, we first use the transformation method by Soleimani et al. [1] as explained in Section 2 to decrease the effect of autocorrelation within each profile. Based on the transformation method, the intercept and the slope in the original model are replaced by the transformed regression parameters. Consequently, the $T^{2}$ statistic is written as follows:

$$
T_{j}^{2}=\mathbf{b}^{T} \boldsymbol{\Sigma}^{-1} \mathbf{b}
$$

where $\mathbf{b}$ and $\boldsymbol{\Sigma}$ are computed using Eqs. (10) and (11), respectively:

$$
\begin{aligned}
& \mathbf{b}=\left[\begin{array}{ll}
\hat{A}_{0 j}^{\prime} & \hat{A}_{1 j}^{\prime}
\end{array}\right]-\left[\begin{array}{ll}
A_{0}^{\prime} & A_{1}^{\prime}
\end{array}\right], \\
& \boldsymbol{\Sigma}=\left(\begin{array}{cc}
\sigma^{2}\left(\frac{1}{n-1}+\frac{\bar{X}^{\prime 2}}{s_{x^{\prime} x^{\prime}}}\right) & -\sigma^{2} \frac{\bar{X}^{\prime}}{s_{x^{\prime} x^{\prime}}} \\
-\sigma^{2} \frac{\bar{X}^{\prime}}{s_{x^{\prime} x^{\prime}}} & \frac{\sigma^{2}}{s_{x^{\prime} x^{\prime}}}
\end{array}\right) .
\end{aligned}
$$

When the process is in-control, the $T^{2}$ statistic follows a central chi-square distribution with two degrees of freedom. Hence, the upper control limit for the $T^{2}$ control chart is UCL $=\chi_{2, \alpha}^{2}$, where $\chi_{2, \alpha}^{2}$ is the 100 $(1-\alpha)$ percentile of the chi-square distribution with two degrees of freedom [1].

\section{The existing estimators to estimate the step change point}

In this section, two estimators in the literature proposed by Kazemzadeh et al. [24] are introduced for estimating the step change point in the parameters of a simple linear profile in the presence of autocorrelation within each profile. They include maximum likelihood and clustering estimators described as follows.

\subsection{The maximum likelihood estimator for the transformed observations}

In this method, to write the likelihood function, first, $y_{i j}^{\prime}$ 's distribution are determined. Since the $u_{i j}$ 's follow the normal distribution, it can be concluded that the $y_{i j}^{\prime}$ has a normal distribution with mean $A_{o}^{\prime}+A_{1}^{\prime} x_{i}^{\prime}$ and variance of $\sigma^{2}$. Therefore, the probability density function of the observations is computed as follows:

$$
f\left(y_{i j}^{\prime}\right)=\frac{1}{\sigma \sqrt{2 \pi}} \exp \left\{-\frac{1}{2 \sigma^{2}}\left(y_{i j}^{\prime}-\left(A_{0}^{\prime}+A_{1}^{\prime} x_{i}^{\prime}\right)\right)^{2}\right\}
$$

where $y_{i j}^{\prime}$ is the response value of $i$ th predictor variable in $j$ th profile. After some times and in unknown profile $\tau$, the process becomes out-of-control and $A_{0}$, $A_{1}$ parameters change as in Eqs. (13) and (14):

$$
\begin{aligned}
& A_{1}^{\prime \prime}=A_{1}+\lambda_{1} \sigma, \\
& A_{0}^{\prime \prime}=A_{0}+\lambda_{2} \sigma .
\end{aligned}
$$

And, until the control chart has not been signaled, the parameters remain in the out-of-control state. Also, it is assumed that the error standard deviation and the autocorrelation coefficient remain fixed during the process.

In order to estimate the unknown parameters using the maximum likelihood estimation method, assuming that the change occurs in the time $\tau$, the likelihood function of model is given as follows:

$$
\begin{aligned}
& L\left(A_{1}^{\prime \prime}, A_{0}^{\prime \prime}, \tau \mid y^{\prime}\right)=\frac{1}{\prod_{j=1}^{T} \prod_{i=1}^{n} \sigma \sqrt{2 \pi}} \\
& . \exp \left\{-\frac{1}{2 \sigma^{2}}\left(\sum_{j=1}^{\tau} \sum_{i=1}^{n}\left(y_{i j}^{\prime}-\left(A_{0}^{\prime}+A_{1}^{\prime} x_{i}^{\prime}\right)\right)^{2}\right.\right. \\
& \left.\left.+\sum_{j=\tau+1}^{T} \sum_{j=1}^{n}\left(y_{i j}^{\prime}-\left(A_{0}^{\prime \prime}(1-\rho)+A_{1}^{\prime \prime} x_{i}^{\prime}\right)\right)^{2}\right)\right\},
\end{aligned}
$$

where $T$ is the number of the first profile which falls out of the upper control limit. In other words, for profiles $j=1,2, \cdots, \tau$, the value of regression parameters is equal to $A_{1}^{\prime}$ and $A_{0}^{\prime}$. And, for profiles $j=\tau+1, \tau+2, \cdots, T$, these values change to $A_{0}^{\prime \prime}(1-\rho)$ and $A_{1}^{\prime \prime}$, respectively. The unknown parameters of the likelihood functions are $\tau, A_{1}^{\prime \prime}$, and $A_{0}^{\prime \prime}$ which should be estimated. The maximum likelihood value of $\tau$ is the value of $\tau$ that maximizes the likelihood function in Eq. (15). Taking the logarithm of Eq. (15), we have:

$$
\operatorname{Ln}\left[L\left(A_{1}^{\prime \prime}, A_{0}^{\prime \prime}, \tau \mid y^{\prime}\right)\right]=\operatorname{Ln}\left(\frac{1}{\prod_{j=1}^{T} \prod_{i=1}^{n} \sigma \sqrt{2 \pi}}\right)
$$




$$
\begin{aligned}
& -\frac{1}{2 \sigma^{2}}\left(\sum_{j=1}^{\tau} \sum_{i=1}^{n}\left(y_{i j}^{\prime}-\left(A_{0}^{\prime}+A_{1}^{\prime} x_{i}^{\prime}\right)\right)^{2}\right. \\
& \left.+\sum_{j=\tau+1}^{T} \sum_{i=1}^{n}\left(y_{i j}^{\prime}-\left(A_{0}^{\prime \prime}(1-\rho)+A_{1}^{\prime \prime} x_{i}^{\prime}\right)\right)^{2}\right) .
\end{aligned}
$$

In Eq. (16), the parameters $A_{1}^{\prime \prime}$ and $A_{0}^{\prime \prime}$ are unknown and should be estimated by taking the partial derivation from Eq. (16) with respect to these parameters. Initially, by taking the partial derivation with respect to $A_{1}^{\prime \prime}$ and $A_{0}^{\prime \prime}$, putting them equal to zero, the values of $\hat{A}_{0}^{\prime \prime}$ and $\hat{A}_{1}^{\prime \prime}$ are estimated. Then, by replacing the obtained values in Eq. (16) and by simplifying it, the change point is obtained using Eq. (17) as follows (the proof is given in Appendix A):

$$
\begin{aligned}
\widehat{\tau}= & \underset{0 \leq \tau<T}{\arg \max }\left[-\frac{1}{2 \sigma^{2}}\left(\sum _ { j = \tau + 1 } ^ { T } \sum _ { i = 1 } ^ { n } \left(\left(y_{i j}^{\prime}\right.\right.\right.\right. \\
& \left.-\left(\hat{A}_{0}^{\prime \prime}(1-\rho)+\hat{A}_{1}^{\prime \prime} x_{i}^{\prime}\right)\right)^{2} \\
& \left.-\sum_{j=1}^{\tau} \sum_{i=1}^{n}\left(y_{i j}^{\prime}-\left(A_{0}^{\prime}+A_{1}^{\prime} x_{i}^{\prime}\right)\right)^{2}\right) .
\end{aligned}
$$

\subsection{The clustering estimator}

The clustering method is the process of dividing data into different groups with the concept of cluster. Thus, a cluster is a series of similar data in a group. We can use the clustering method to estimate the change point because we can separate the observations into two clusters: in-control and out-of-control [15].

To use the clustering method, initially, the autocorrelated observations are transformed and the effect of autocorrelation is eliminated. Now, the clustering method is used on the transformed observations and the change point is estimated. The structure of the clustering method is described as follows:

Suppose that an out-of-control signal is notified by the control chart at the time $T$. We are intended to find $\tau$, the real time of the change, where the parameters of profile change from $A_{0}$ and $A_{1}$ to $A_{0}^{\prime \prime}$ and $A_{1}^{\prime \prime}$, respectively. Hence, two clusters including incontrol and out-of-control clusters are considered. All observations before $\tau$ belong to the in-control cluster, and the observations after $\tau$ are placed in out-ofcontrol cluster. To estimate the change point, first, an index is suggested. The index is computed for all possible combinations of observation's assignment to each of the in-control and out-of-control clusters. The change point is the point in which the proposed index is optimized. The clustering index, considering Ghazanfari et al. [15], is defined as follows, such that the lowest values of the variation within each cluster are obtained:

$$
\begin{aligned}
& \mathrm{SSW}=\mathrm{SSW}_{\mathrm{in}}+\mathrm{SSW}_{\text {out }}, \\
& \mathrm{SSW}=\sum_{j=1}^{\tau} \mathbf{M}^{\prime} \boldsymbol{\Sigma}_{0}^{-1} \mathbf{M}+\sum_{j=\tau+1}^{T} \mathbf{N}^{\prime} \boldsymbol{\Sigma}_{0}^{-1} \mathbf{N},
\end{aligned}
$$

where:

$$
\begin{aligned}
& \mathbf{M}=\left[\left(\hat{A}_{0 j}^{\prime} \hat{A}_{1 j}^{\prime}\right)-\left(A_{0}^{\prime} A_{1}^{\prime}\right)\right], \\
& \mathbf{N}=\left[\left(\hat{A}_{0 j}^{\prime} \hat{A}_{1 j}^{\prime}\right)-C_{\text {out }}\right]
\end{aligned}
$$

and:

$$
C_{\text {out }}=\left(\sum_{j=\tau+1}^{T} \frac{\hat{A}_{0 j}^{\prime}}{(T-\tau)} \sum_{j=\tau+1}^{T} \frac{\hat{A}_{1 j}^{\prime}}{(T-\tau)}\right) .
$$

$C_{\text {out }}$ is considered as the center of the out-of-control cluster. Also, $\sum_{0}^{-1}$ is the inverse of covariance matrix of the process which was defined in Eq. (11). SW in and $S S W_{\text {out }}$ show the variation of in-control and out-ofcontrol clusters, respectively. When this index has the lowest value, it means that observations are assigned to the most appropriate cluster. Hence, the change point is the $\tau$ in which this index is minimized:

$$
\widehat{\tau}=\underset{0 \leq \tau<T}{\arg } \min \{\mathrm{SSW}\}
$$

\section{Proposed step change point estimator}

In this section, we propose another method to deal with the autocorrelation effect of observations within each profile which seems to give more accurate results compared to the transformation and the clustering estimators. In this method, instead of eliminating the autocorrelation effect of observations using the explained transformation method, we obtain the joint probability distribution of the autocorrelated observations, and then we use the maximum likelihood estimation method to estimate the step change point.

Timmer et al. [26] found the joint probability density function of the AR(1) observations. They used the following model for the first-order autoregressive process:

$$
\varepsilon_{i j}=\rho \varepsilon_{(i-1) j}+\delta+u_{i j},
$$

where $u_{i j} \sim N\left(0, \sigma^{2}\right)$ is a normal random variable with the mean 0 and the variance $\sigma^{2}$.

In this parameterization of the $\operatorname{AR}(1)$ model, $\delta$ is the location parameter. The autocorrelation coefficient is $\rho$, and $\sigma^{2}$ is the variance of the (Gaussian) white noise process. The steady-state expected value of $\varepsilon_{i j}$ is $E\left(\varepsilon_{i j}\right)=\frac{\delta}{1-\rho}$. They presented the joint 
probability density function (pdf) for the $\mathrm{AR}(1)$ process. Accordingly, they developed the joint probability density function of $\mathrm{AR}(1)$ autocorrelation observations as follows:

$$
\begin{aligned}
& f\left(\varepsilon_{1 j}, \varepsilon_{2 j}, \cdots, \varepsilon_{n j}\right)=\left(2 \pi \sigma^{2}\right)^{-n / 2}\left(1-\rho^{2}\right)^{1 / 2} \\
& \quad \times \exp \left[-\frac{1}{2 \sigma^{2}}\left\{\left(1-\rho^{2}\right)\left(\varepsilon_{1 j}-\frac{\delta}{1-\rho}\right)^{2}\right.\right. \\
& \left.\left.\quad+\sum_{i=2}^{n}\left(\varepsilon_{i j}-\delta-\rho \varepsilon_{(i-1) j}\right)^{2}\right\}\right] .
\end{aligned}
$$

Based on Eqs. (1) and (2), the following Eq. is obtained:

$$
y_{i j}-\left(A_{0}+A_{1} x_{i}\right)=\rho\left(y_{(i-1) j}-\left(A_{0}+A_{1} x_{i-1}\right)\right)+u_{i j} \text {. }
$$

As a result:

$$
y_{i j}=\rho y_{(i-1) j}+\left[\left(A_{0}+A_{1} x_{i}\right)-\rho\left(A_{0}+A_{1} x_{i-1}\right)\right]+u_{i j} .
$$

Eq. (24) is similar to Eq. (21) developed by Timmer et al. [26], where $\delta_{i}=\left[\left(A_{0}+A_{1} x_{i}\right)-\rho\left(A_{0}+A_{1} x_{i-1}\right)\right]$. Now, we can show the joint probability density function of the observations based on Eqs. (22) and (24) as follows:

$$
\begin{aligned}
& f\left(y_{1 j}, y_{2 j}, \cdots, y_{n j}\right)=\left(2 \pi \sigma^{2}\right)^{-n / 2}\left(1-\rho^{2}\right)^{1 / 2} \\
& \quad \times \exp \left[-\frac{1}{2 \sigma^{2}}\left\{\left(1-\rho^{2}\right)\left(y_{1 j}-\left(A_{0}+A_{1} x_{1}\right)\right)^{2}\right.\right. \\
& \left.\quad+\sum_{i=2}^{n}\left(y_{i j}-\delta_{i}-\rho y_{(i-1) j)^{2}}\right\}\right] \\
& \text { for } j=1, \cdots, T .
\end{aligned}
$$

In Eq. (25), $A_{0}$ and $A_{1}$ are the regression parameters values under the in-control state; $y_{i j}$ 's are the response observations; $\rho$ is the coefficient of autocorrelation; and $n$ shows the number of levels of independent variables. Based on the probability density function of the autocorrelated response observations, we compute the likelihood function and estimate the step change point. The likelihood function generally is obtained as multiply of the joint probability density functions of the response variables for each profile. Accordingly, using Eq. (25), the likelihood function for the simple linear profile model with the AR(1) structure in the residuals is developed as follows:

$$
\begin{aligned}
L\left(A_{0}, A_{1}, \tau \mid y\right)= & \prod_{j=1}^{\tau} f\left(y_{1 j}, y_{2 j}, \cdots, y_{n j}\right) \\
& \prod_{i=\tau+1}^{T} f\left(y_{1 j}, y_{2 j}, \cdots, y_{n j}\right) .
\end{aligned}
$$

As a result:

$$
\begin{aligned}
& L\left(A_{0}, A_{1}, \tau \mid y\right)=\prod_{j=1}^{\tau}\left\{\left(2 \pi \sigma^{2}\right)^{-n / 2}\left(1-\rho^{2}\right)^{1 / 2}\right. \\
& \times \exp \left[-\frac{1}{2 \sigma^{2}}\left\{\left(1-\rho^{2}\right)\left(y_{1 j}-\left(A_{0}+A_{1} x_{1}\right)\right)^{2}\right.\right. \\
& \left.\left.\left.+\sum_{i=2}^{n}\left(y_{i j}-\delta_{i}-\rho y_{(i-1) j}\right)^{2}\right\}\right]\right\} \\
& \quad \times \prod_{j=\tau+1}^{T}\left\{\left(2 \pi \sigma^{2}\right)^{-n / 2}\left(1-\rho^{2}\right)^{1 / 2}\right. \\
& \quad \times \exp \left[-\frac{1}{2 \sigma^{2}}\left\{\left(1-\rho^{2}\right)\left(y_{1 j}-\left(A^{\prime \prime}{ }_{0}+A^{\prime \prime}{ }_{1} x_{1}\right)\right)^{2}\right.\right. \\
& \left.\left.+\sum_{i=2}^{n}\left(y_{i j}-\delta_{i}^{\prime}-\rho y_{(i-1) j}\right)^{2}\right\}\right] .
\end{aligned}
$$

Since the likelihood function and its logarithm have the same extremum point, so we have:

$$
\begin{aligned}
\log ( & \left.L\left(A_{0}, A_{1}, \tau \mid y\right)\right)=\sum_{j=1}^{\tau} \log \left(\left(2 \pi \sigma^{2}\right)^{-n / 2}\left(1-\rho^{2}\right)^{1 / 2}\right) \\
& +\sum_{j=1}^{\tau}\left[-\frac{1}{2 \sigma^{2}}\left\{\left(1-\rho^{2}\right)\left(y_{1 j}-\left(A_{0}+A_{1} x_{1}\right)\right)^{2}\right.\right. \\
+ & \left.\left.\sum_{i=2}^{n}\left(y_{i j}-\delta_{i}-\rho y_{(i-1) j}\right)^{2}\right\}\right] \\
+ & \sum_{j=\tau+1}^{T} \log \left(\left(2 \pi \sigma^{2}\right)^{-n / 2}\left(1-\rho^{2}\right)^{1 / 2}\right) \\
+ & \sum_{j=\tau+1}^{T}\left[-\frac{1}{2 \sigma^{2}}\left\{\left(1-\rho^{2}\right)\left(y_{1 j}-\left(A_{0}^{\prime \prime}+A_{1}^{\prime \prime} x_{1}\right)\right)^{2}\right.\right. \\
+ & \left.\left.\sum_{i=2}^{n}\left(y_{i j}-\delta_{i}^{\prime}-\rho y_{(i-1) j}\right)^{2}\right\}\right] .
\end{aligned}
$$

It is assumed that the regression parameters of the AR(1) autocorrelated simple linear profile are out-ofcontrol after the time $\tau$. This means that the regression parameters are $A_{0}$ and $A_{1}$ for profiles $1,2, \cdots, \tau$ and $A_{0}^{\prime \prime}$ and $A_{1}^{\prime \prime}$ for profiles $\tau+1, \tau+2, \cdots, T$, where $T$ is when an out-of-control signal appears on the control chart. In Eq. (28), the parameters $\tau, A_{0}^{\prime \prime}$, and $A_{1}^{\prime \prime}$ are unknown and they should be estimated. At first, the parameters $A_{0}^{\prime \prime}$ and $A_{1}^{\prime \prime}$ are estimated, and then the 
estimates are replaced in the logarithm of the likelihood function. Eventually, the change point estimator is obtained as follows (proof is given in Appendix B):

$$
\begin{aligned}
\hat{\tau}=\underset{0 \leq \tau<T}{\arg \max } & \left\{\sum _ { j = \tau + 1 } ^ { T } \left[-\frac{1}{2 \sigma^{2}}\left\{\left(1-\rho^{2}\right)\left(y_{1 j}-\left(\hat{A}_{0}^{\prime \prime}+\hat{A}_{1}^{\prime \prime} x_{1}\right)\right)^{2}\right.\right.\right. \\
& \left.\left.+\sum_{i=2}^{n}\left(y_{i j}-\hat{\delta}_{i}^{\prime}-\rho y_{(i-1) j}\right)^{2}\right\}\right] \\
& -\sum_{j=\tau+1}^{T}\left[-\frac{1}{2 \sigma^{2}}\left\{\left(1-\rho^{2}\right)\left(y_{1 j}-\left(A_{0}+A_{1} x_{1}\right)\right)^{2}\right.\right. \\
& \left.\left.\left.+\sum_{i=2}^{n}\left(y_{i j}-\delta_{i}-\rho y_{(i-1) j}\right)^{2}\right\}\right]\right\} .
\end{aligned}
$$

The change point estimator $(\hat{\tau})$ is the value that maximizes the above equation. In the next section, performance of the proposed estimator is evaluated through simulation studies.

\section{Simulation studies}

In this section, we evaluate the performance of the proposed estimator using Monte Carlo simulation, and the results are compared with the performance of the two existing estimators in the literature. To do so, it is assumed that the $\operatorname{AR}(1)$ autocorrelated simple linear profile model under the in-control state is as $y_{i j}=3+2 x_{i}+\varepsilon_{i j}$, where the residuals have $\mathrm{AR}(1)$ structure and are modeled as $\varepsilon_{i j}=\rho \varepsilon_{(i-1) j}+u_{i j}$. $u_{i j}$ 's are the normal random variables with mean 0 and variance 1 . Four levels for explanatory variable are considered as follows, which are the same in all profiles:

$$
\mathbf{x}=[2468]
$$

The $T^{2}$ control chart with the probability of false alarm equal to 0.005 is used to monitor the profiles. Also, it is assumed that the real change point value is equal to 50. The simulation procedure is such that initially, 50 vectors for the response variable are generated using the autocorrelated simple linear profile model under in-control state. Then, the transformation method is implemented on the explanatory and the response variables. According to the transformed variables, the regression parameters are estimated for each of the 50 profiles, and then $T_{j}^{2}$ 's statistics for 50 profiles are calculated using the values of estimated parameters. During the generation of the 50 profiles, the statistics related to some profiles may fall out of the UCL.
These profiles are considered as false alarms because we assumed that the first 50 observations are generated based on the in-control model. So, if there is a statistic that is out-of-control at time $t<\tau$, it is considered as a false alarm. After the 51 th point, the regression parameters of the model $\left(A_{0}\right.$ and $A_{1}$ ) change to $A_{0}+\lambda_{1} \sigma$ and $A_{1}+\lambda_{2} \sigma$. Consequently, the new model whose parameters are out-of-control is obtained as follows:

$$
\begin{aligned}
& y_{i j}=\left(3+\lambda_{1}\right)+\left(2+\lambda_{2}\right) x_{i}+\varepsilon_{i j}, \\
& k=2(T-\tau)\left[\left(1-\rho^{2}\right) x_{1}+(\rho-1) \sum_{i=2}^{n}\left(\rho x_{i-1}-x_{i}\right)\right] .
\end{aligned}
$$

After time of 50, the profiles are generated based on the above out-of-control model. The generation of profiles last as long as the $T^{2}$ control chart warn that the process is out-of-control. At this moment, the generation of observations is ceased and the proposed estimator is applied for all obtained profiles. To compute the change point by the proposed estimator, the maximum likelihood method is used for the autocorrelated observations. This procedure is repeated 10,000 times, and finally, the mean of all the obtained change points from all iterations is reported as the estimated change point.

To evaluate the accuracy and precision of the proposed estimator, proportion of the times' numbers which the estimated change point place on the distance of maximum $i(i=1,2, \cdots, 15)$ from the real change point is computed as $p(|\hat{\tau}-\tau| \leq i)$. And, based on the obtained probabilities, it is clarified that how far the estimated change point from the real change point is. Finally, the obtained results are compared with the results of the two existing estimators in the literature under the weak autocorrelation coefficient $(0.1)$ and the strong autocorrelation coefficient $(0.9)$. The results of these comparisons are reported in Tables 1 to 4 .

Table 1 shows the simulation results of three estimators under the autocorrelation coefficient of 0.1 between the observations within each profile, considering various shifts in the $A_{1}$ parameter. The first three rows of Table 1 demonstrate the mean of estimated change points by three estimators. (For better illustration, the results are also depicted in Figure 1.) The results show better accuracy of the proposed estimator rather than the other estimator especially in large shifts. However, in very small shifts in the slope, the accuracy of the clustering estimator is roughly better than the proposed estimator. In the next rows of the table, the standard errors of change point estimates as well as the probability values for $i=1,2, \cdots, 15$ by three estimators are reported. The smaller values of standard error as well as the larger probability values by the proposed estimator 
Table 1. The accuracy and precision of estimators under shift in the slope from $A_{1}$ to $A_{1}+\lambda_{1} \sigma$ and $\rho=0.9$ when $\tau=50$.

\begin{tabular}{lcccccc}
\hline & $\mathbf{0 . 0 8}$ & $\mathbf{0 . 0 6}$ & $\mathbf{0 . 0 4}$ & $\mathbf{0 . 0 2}$ & $\mathbf{0 . 0 1}$ & $\boldsymbol{\lambda}_{\mathbf{1}}$ \\
\hline The proposed estimator & 49.97 & 51.02 & 54.54 & 65.54 & 72.19 & $\overline{\hat{\boldsymbol{\tau}}}$ \\
MLE estimator & 49.95 & 51.43 & 55.86 & 66.14 & 74.79 & \\
Clustering estimator & 46.5 & 48.65 & 50.88 & 61.14 & 66.83 & \\
The proposed estimator & 0.14 & 0.18 & 0.29 & 0.45 & 0.57 & $\mathbf{s e}$ \\
MLE estimator & 0.14 & 0.19 & 0.30 & 0.49 & 0.59 & \\
Clustering estimator & 0.20 & 0.25 & 0.35 & 0.48 & 0.59 & \\
The proposed estimator & 0.21 & 0.12 & 0.07 & 0.04 & 0.02 & \\
MLE estimator & 0.18 & 0.11 & 0.06 & 0.03 & 0.02 & $p(|\widehat{\boldsymbol{\tau}}-\boldsymbol{\tau}| \leq 0)$ \\
Clustering estimator & 0.13 & 0.09 & 0.04 & 0.03 & 0.02 & \\
The proposed estimator & 0.37 & 0.25 & 0.16 & 0.08 & 0.06 & \\
MLE estimator & 0.34 & 0.23 & 0.15 & 0.08 & 0.05 & $p(|\widehat{\boldsymbol{\tau}}-\boldsymbol{\tau}| \leq 1)$ \\
Clustering estimator & 0.26 & 0.19 & 0.13 & 0.09 & 0.05 & \\
The proposed estimator & 0.49 & 0.35 & 0.23 & 0.12 & 0.09 & \\
MLE estimator & 0.45 & 0.34 & 0.22 & 0.11 & 0.08 & $p(|\widehat{\boldsymbol{\tau}}-\boldsymbol{\tau}| \leq 2)$ \\
Clustering estimator & 0.41 & 0.28 & 0.19 & 0.11 & 0.09 & \\
The proposed estimator & 0.58 & 0.43 & 0.29 & 0.16 & 0.12 & $p(|\widehat{\boldsymbol{\tau}}-\boldsymbol{\tau}| \leq 3)$ \\
MLE estimator & 0.56 & 0.41 & 0.27 & 0.14 & 0.11 & \\
Clustering estimator & 0.48 & 0.36 & 0.25 & 0.15 & 0.12 & \\
The proposed estimator & 0.64 & 0.50 & 0.34 & 0.20 & 0.15 & \\
MLE estimator & 0.61 & 0.48 & 0.31 & 0.18 & 0.13 & $p(|\widehat{\boldsymbol{\tau}}-\boldsymbol{\tau}| \leq 4)$ \\
Clustering estimator & 0.54 & 0.41 & 0.28 & 0.19 & 0.14 & \\
The proposed estimator & 0.92 & 0.84 & 0.66 & 0.48 & 0.40 & \\
Clustering estimator & 0.83 & 0.74 & 0.59 & 0.46 & 0.35 & \\
\hline & 0.90 & 0.81 & 0.63 & 0.43 & 0.34 & $p(|\widehat{\boldsymbol{\tau}}-\boldsymbol{\tau}| \leq 5)$ \\
\hline
\end{tabular}

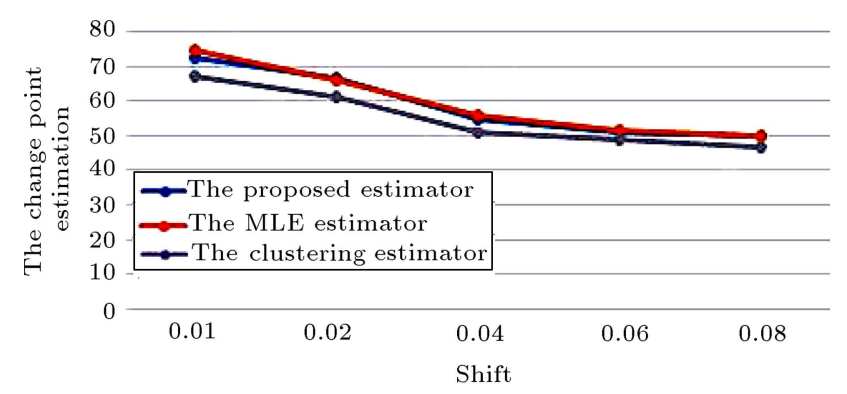

Figure 1. The mean of the change point estimates under different shifts in the parameter $A_{1}$ by three estimators when the real change point is equal to 50 and the autocorrelation coefficient is 0.1 .

rather than the other existing estimators confirm better precision of the proposed estimator in estimating the change point.

Table 2 indicates the simulation results of the proposed estimator under the autocorrelation coefficient of 0.9 , and the results are compared with two other estimators under different shifts in $A_{1}$ regression parameter. The first three rows of Table 2 illustrate that the estimations obtained by the proposed estimator are closer to the real change point value compared with the other two estimators. (See Figure 2 for elaborating the results regarding the mean of change point estimates using three methods.) The comparison of the estimators based on the obtained standard error and probability values confirm the better precision of the proposed estimator rather than the other estimators in most of the shifts considered.

Table 3 expresses the simulation results of three estimators when shifts occur in $A_{0}$ regression parameter, and also there is the autocorrelation between the observations within each profile with autocorrelation coefficient of 0.1 . The first three rows of the table indicate the mean of change point estimates by three estimators. (Refer to Figure 3 for better understanding 
Table 2. The accuracy and precision of estimators under shift in the slope from $A_{1}$ to $A_{1}+\lambda_{1} \sigma$ and $\rho=0.9$ when $\tau=50$.

\begin{tabular}{lcccccc}
\hline & $\mathbf{0 . 0 8}$ & $\mathbf{0 . 0 6}$ & $\mathbf{0 . 0 4}$ & $\mathbf{0 . 0 2}$ & $\mathbf{0 . 0 1}$ & $\boldsymbol{\lambda}_{\mathbf{2}}$ \\
\hline The proposed estimator & 52.18 & 56.54 & 63.04 & 66.16 & 72.49 & $\overline{\hat{\boldsymbol{\tau}}}$ \\
MLE estimator & 54.82 & 58.92 & 65.54 & 71.43 & 76.09 & \\
Clustering estimator & 53.90 & 58.18 & 61.26 & 68.01 & 74.03 & \\
The proposed estimator & 0.28 & 0.33 & 0.41 & 0.49 & 0.58 & \\
MLE estimator & 0.37 & 0.44 & 0.53 & 0.59 & 0.66 & $\boldsymbol{s e} \overline{\boldsymbol{\tau}}$ \\
Clustering estimator & 0.38 & 0.42 & 0.48 & 0.53 & 0.61 & \\
The proposed estimator & 0.08 & 0.06 & 0.03 & 0.02 & 0.02 & \\
MLE estimator & 0.05 & 0.03 & 0.02 & 0.01 & 0.01 & $p(|\widehat{\boldsymbol{\tau}}-\boldsymbol{\tau}| \leq 0)$ \\
Clustering estimator & 0.06 & 0.04 & 0.03 & 0.02 & 0.02 & \\
The proposed estimator & 0.12 & 0.10 & 0.09 & 0.07 & 0.05 & \\
MLE estimator & 0.10 & 0.07 & 0.06 & 0.04 & 0.04 & $p(|\widehat{\boldsymbol{\tau}}-\boldsymbol{\tau}| \leq 1)$ \\
Clustering estimator & 0.11 & 0.10 & 0.06 & 0.04 & 0.04 & \\
The proposed estimator & 0.19 & 0.17 & 0.15 & 0.09 & 0.08 & \\
MLE estimator & 0.15 & 0.12 & 0.09 & 0.07 & 0.06 & $p(|\widehat{\boldsymbol{\tau}}-\boldsymbol{\tau}| \leq 2)$ \\
Clustering estimator & 0.14 & 0.14 & 0.13 & 0.12 & 0.07 & \\
The proposed estimator & 0.23 & 0.21 & 0.18 & 0.14 & 0.11 & $p(|\widehat{\boldsymbol{\tau}}-\boldsymbol{\tau}| \leq 3)$ \\
MLE estimator & 0.20 & 0.15 & 0.12 & 0.10 & 0.09 & \\
Clustering estimator & 0.21 & 0.20 & 0.18 & 0.17 & 0.09 & \\
The proposed estimator & 0.29 & 0.25 & 0.22 & 0.17 & 0.14 & \\
MLE estimator & 0.28 & 0.21 & 0.17 & 0.14 & 0.13 & $p(|\widehat{\boldsymbol{\tau}}-\boldsymbol{\tau}| \leq 4)$ \\
Clustering estimator & 0.29 & 0.25 & 0.21 & 0.17 & 0.14 & \\
The proposed estimator & 0.66 & 0.59 & 0.44 & 0.39 & 0.35 & \\
MLE estimator & 0.54 & 0.46 & 0.39 & 0.34 & 0.33 & $p(|\widehat{\boldsymbol{\tau}}-\boldsymbol{\tau}| \leq 5)$ \\
Clustering estimator & 0.64 & 0.59 & 0.54 & 0.51 & 0.35 & \\
\hline
\end{tabular}

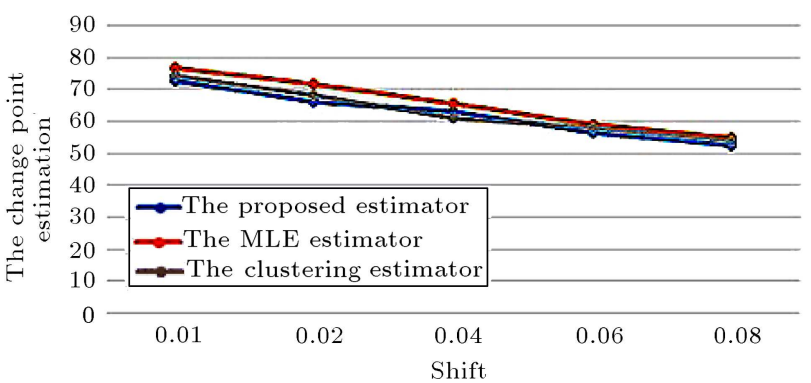

Figure 2. The mean of the change point estimates under different shifts in the parameter $A_{1}$ by three estimators when the real change point is equal to 50 and the autocorrelation coefficient is 0.9 .

of the results.) The results show the better accuracy of the proposed estimator in all shifts considered. The standard error of change point estimates and the probabilities of locating the change point estimates at different intervals from the real change point are

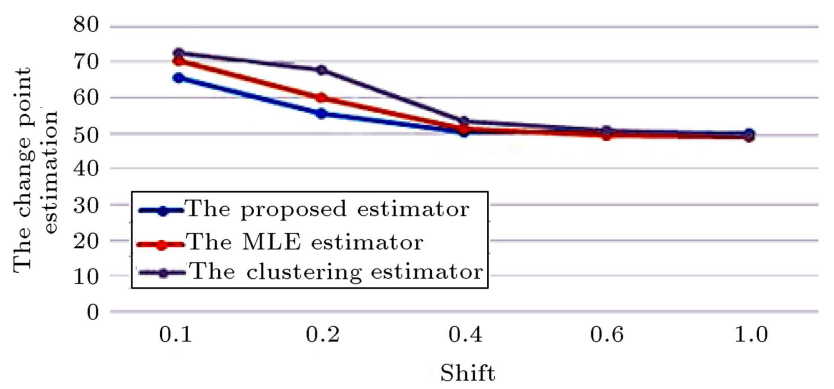

Figure 3. The mean of the change point estimates under different shifts in the parameter $A_{0}$ by three estimators when the real change point is equal to 50 and the autocorrelation coefficient is 0.1 .

also reported in the next rows of Table 3 . As shown in this table, the probabilities of estimating the real change point in different distances from the real change in the proposed estimator is larger than the obtained proportions by the other two estimators 
Table 3. The accuracy and precision of estimators under shift in the slope from $A_{0}$ to $A_{0}+\lambda_{1} \sigma$ and $\rho=0.1$ when $\tau=50$.

\begin{tabular}{lcccccc}
\hline & $\mathbf{1}$ & $\mathbf{0 . 6}$ & $\mathbf{0 . 4}$ & $\mathbf{0 . 2}$ & $\mathbf{0 . 1}$ & $\boldsymbol{\lambda}_{\mathbf{1}}$ \\
\hline The proposed estimator & 50.14 & 50.26 & 50.60 & 55.59 & 65.80 & $\overline{\hat{\boldsymbol{\tau}}}$ \\
MLE estimator & 49.26 & 49.58 & 51.14 & 60.21 & 70.54 & \\
Clustering estimator & 49.55 & 50.68 & 53.50 & 67.78 & 72.74 & \\
The proposed estimator & 0.05 & 0.10 & 0.15 & 0.31 & 0.48 & \\
MLE estimator & 0.07 & 0.12 & 0.18 & 0.36 & 0.52 & $\boldsymbol{s e} \overline{\boldsymbol{\tau}}$ \\
Clustering estimator & 0.06 & 0.11 & 0.19 & 0.38 & 0.51 & \\
The proposed estimator & 0.10 & 0.09 & 0.07 & 0.03 & 0.02 & \\
MLE estimator & 0.09 & 0.07 & 0.05 & 0.03 & 0.02 & $p(|\widehat{\boldsymbol{\tau}}-\boldsymbol{\tau}| \leq 0)$ \\
Clustering estimator & 0.07 & 0.04 & 0.03 & 0.02 & 0.02 & \\
The proposed estimator & 0.71 & 0.45 & 0.28 & 0.12 & 0.06 & \\
MLE estimator & 0.70 & 0.43 & 0.26 & 0.11 & 0.05 & $p(|\widehat{\boldsymbol{\tau}}-\boldsymbol{\tau}| \leq 1)$ \\
Clustering estimator & 0.70 & 0.41 & 0.24 & 0.09 & 0.06 & \\
The proposed estimator & 0.86 & 0.62 & 0.41 & 0.18 & 0.10 & \\
MLE estimator & 0.83 & 0.55 & 0.36 & 0.17 & 0.09 & $p(|\widehat{\boldsymbol{\tau}}-\boldsymbol{\tau}| \leq 2)$ \\
Clustering estimator & 0.81 & 0.53 & 0.34 & 0.13 & 0.09 & \\
The proposed estimator & 0.93 & 0.72 & 0.50 & 0.24 & 0.13 & \\
MLE estimator & 0.88 & 0.64 & 0.44 & 0.21 & 0.11 & $p(|\widehat{\boldsymbol{\tau}}-\boldsymbol{\tau}| \leq 3)$ \\
Clustering estimator & 0.88 & 0.62 & 0.41 & 0.17 & 0.12 & \\
The proposed estimator & 0.95 & 0.78 & 0.57 & 0.29 & 0.17 & \\
MLE estimator & 0.91 & 0.70 & 0.51 & 0.26 & 0.16 & $p(|\widehat{\boldsymbol{\tau}}-\boldsymbol{\tau}| \leq 4)$ \\
Clustering estimator & 0.91 & 0.69 & 0.46 & 0.20 & 0.17 & \\
The proposed estimator & 0.99 & 0.96 & 0.88 & 0.63 & 0.45 & \\
MLE estimator & 0.98 & 0.94 & 0.84 & 0.55 & 0.42 & $p(|\widehat{\boldsymbol{\tau}}-\boldsymbol{\tau}| \leq 5)$ \\
Clustering estimator & 0.98 & 0.93 & 0.82 & 0.50 & 0.36 & \\
\hline
\end{tabular}

in the literature. Also, the standard error of the estimates by the proposed estimator is smaller than the other estimators. These two criteria confirm the better precision of the proposed estimator.

Table 4 includes the results of the change point estimation by three estimators when the shifts occur in $A_{0}$ regression parameter, and there is the autocorrelation coefficient of 0.9 between the observations within each profile. The results show the better accuracy of the proposed estimator compared to the accuracy of the two other estimators. The mean values of the change point estimates by three estimators in this case are also depicted in Figure 4. The standard error values as well as probability values, reported in the next rows of Table 4, also confirm precision of the proposed estimator with respect to the other estimators.

Generally, the proposed estimator performs better than the other two methods in terms of both accuracy and precision criteria.

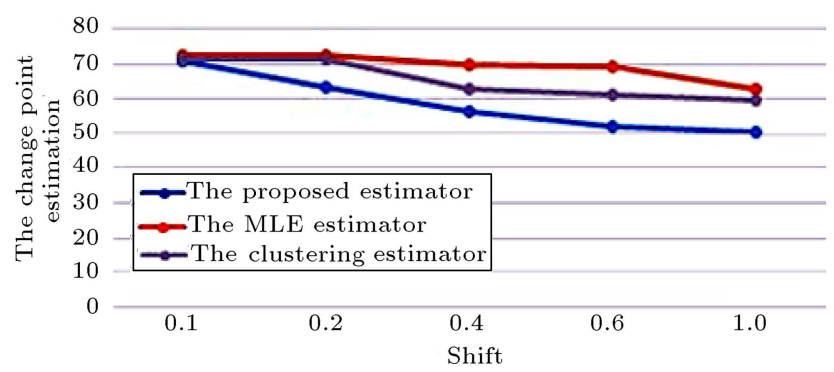

Figure 4. The mean of the change point estimates under different shifts in the parameter $A_{0}$ by three estimators when the real change point is equal to 50 and the autocorrelation coefficient is 0.9 .

\section{Case study}

In this section, a real data based on the findings of Soleimani et al. [1] is used to demonstrate the performance of the proposed estimator in practical applications. In this case study, there are 10 apple 
Table 4. The accuracy and precision of estimators under shift in the slope from $A_{0}$ to $A_{0}+\lambda_{1} \sigma$ and $\rho=0.9$ when $\tau=50$.

\begin{tabular}{|c|c|c|c|c|c|c|}
\hline & 1 & 0.6 & 0.4 & 0.2 & 0.1 & $\lambda_{2}$ \\
\hline The proposed estimator & 50.59 & 52.25 & 56.53 & 63.28 & 70.83 & \\
\hline MLE estimator & 62.78 & 69.05 & 69.95 & 72.29 & 72.67 & $\overline{\hat{\tau}}$ \\
\hline Clustering estimator & 59.31 & 61.11 & 62.82 & 71.11 & 71.26 & \\
\hline The proposed estimator & 0.11 & 0.24 & 0.32 & 0.42 & 0.55 & \\
\hline MLE estimator & 0.51 & 0.59 & 0.6327 & 0.633 & 0.639 & $s e_{\overline{\hat{\tau}}}$ \\
\hline Clustering estimator & 0.502 & 0.56 & 0.57 & 0.601 & 0.613 & \\
\hline The proposed estimator & 0.09 & 0.08 & 0.03 & 0.02 & 0.01 & \\
\hline MLE estimator & 0.06 & 0.04 & 0.02 & 0.01 & 0.01 & $p(|\widehat{\boldsymbol{\tau}}-\boldsymbol{\tau}| \leq 0)$ \\
\hline Clustering estimator & 0.07 & 0.04 & 0.04 & 0.04 & 0.03 & \\
\hline The proposed estimator & 0.38 & 0.22 & 0.13 & 0.07 & 0.05 & \\
\hline MLE estimator & 0.11 & 0.06 & 0.05 & 0.04 & 0.04 & $p(|\widehat{\boldsymbol{\tau}}-\boldsymbol{\tau}| \leq 1)$ \\
\hline Clustering estimator & 0.12 & 0.09 & 0.07 & 0.06 & 0.04 & \\
\hline The proposed estimator & 0.58 & 0.30 & 0.20 & 0.11 & 0.08 & \\
\hline MLE estimator & 0.15 & 0.09 & 0.07 & 0.06 & 0.06 & $p(|\widehat{\boldsymbol{\tau}}-\boldsymbol{\tau}| \leq 2)$ \\
\hline Clustering estimator & 0.18 & 0.16 & 0.14 & 0.13 & 0.07 & \\
\hline The proposed estimator & 0.66 & 0.36 & 0.25 & 0.16 & 0.12 & \\
\hline MLE estimator & 0.17 & 0.12 & 0.10 & 0.09 & 0.09 & $p(|\widehat{\boldsymbol{\tau}}-\boldsymbol{\tau}| \leq 3)$ \\
\hline Clustering estimator & 0.20 & 0.18 & 0.18 & 0.17 & 0.10 & \\
\hline The proposed estimator & 0.73 & 0.41 & 0.30 & 0.22 & 0.15 & \\
\hline MLE estimator & 0.21 & 0.17 & 0.13 & 0.11 & 0.11 & $p(|\widehat{\boldsymbol{\tau}}-\boldsymbol{\tau}| \leq 4)$ \\
\hline Clustering estimator & 0.24 & 0.21 & 0.20 & 0.18 & 0.12 & \\
\hline The proposed estimator & 0.92 & 0.78 & 0.63 & 0.49 & 0.41 & \\
\hline MLE estimator & 0.43 & 0.39 & 0.35 & 0.34 & 0.33 & $p(|\widehat{\boldsymbol{\tau}}-\boldsymbol{\tau}| \leq 5)$ \\
\hline Clustering estimator & 0.50 & 0.45 & 0.43 & 0.39 & 0.37 & \\
\hline
\end{tabular}

trees and 25 apples are chosen randomly from each tree. Analysis is done for the apples whose initial diameter is larger than 2.75 inch. Consequently, from the existing 250 apples, only 80 apples are held over. Each apple diameter is measured every two weeks in 12 weeks. Thus, there are 6 different measurements for each 80 apples that the measurements of each apple constitute a simple linear profile structure. The analysis approves the $\mathrm{AR}(1)$ autocorrelation structure between observations of each profile. In this example, the relationship between the diameter and time is an important quality characteristic, which should be monitored during the time. Hence, a simple linear profile is generated for each apple, and Hoteling method is monitored by $T^{2}$. Finally, the change point is estimated by the proposed estimator, and results are compared with the two estimators in the literature. The data analysis based on calculations of the Phase I provides the values $0.02,2.68$, and 0.7 for the slope, intercept, and the autocorrelation coefficient, respectively. The value of 0.0004 is obtained for variance of $a_{i j} \mathrm{~s}$ as well. The obtained equation is as $y_{i j}=2.68+0.02 x_{i}+\varepsilon_{i j}$ where $\varepsilon_{i j}=0.7 \varepsilon_{(i-1) j}+a_{i j}$ and it has the $\operatorname{AR}(1)$ structure. $a_{i j}$ s follow the standard normal distribution with mean and variance of 0 and 0.0004 , respectively. After applying the transformation method to the observations and monitoring of the profiles by the $T^{2}$ Hoteling control chart, we generate the shifts in $A_{1}$ regression parameter from sample 26 , and generate the observations until a signal is taken by the control chart. Then, the change point is estimated by the proposed method as well as the estimators in the literature. As illustrated in Figure 5, the accuracy of the proposed estimator is better than the ones in the literature, because it estimates the change point exactly at time 25 which is the real change point. 


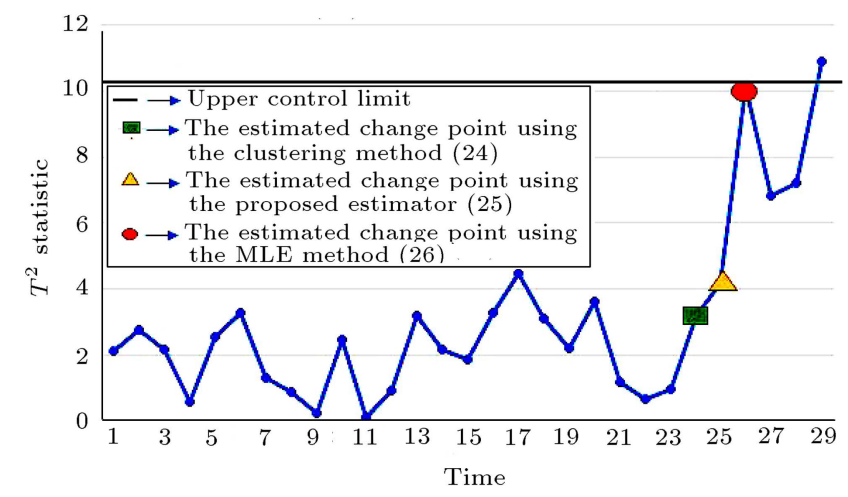

Figure 5. The $T^{2}$ control chart for data of the apple trees under shift in the slope from $A_{1}$ to $A_{1}+0.009 \sigma$ by the proposed estimator and two methods in the literature (real change point is $\tau=25$ ).

\section{Conclusion and future researches}

In this paper, we proposed a new estimator to estimate the step change point in Phase II monitoring of AR(1) autocorrelated simple linear profiles when the autocorrelation structure of the observations within each profile is $\operatorname{AR}(1)$. In the proposed estimator, we used the joint probability density function of the $\operatorname{AR}(1)$ autocorrelated observations to develop the MLE change point estimator for original observations instead of developing the estimator for transformed observations after eliminating the effect of autocorrelation. This is the main novelty of the paper. Performance of the proposed estimator was evaluated through simulation, and the results were compared with the performance of two estimators in the literature. The results indicated that under both autocorrelation coefficients of 0.9 and 0.1 and in most shifts in the regression parameters, the proposed estimator outperforms the other estimators. However, in the case of shift in parameter $A_{1}$, in four initial shifts, the clustering estimator outperforms the proposed estimator. One can generalize the type of profile to the multivariate linear profiles rather than the simple linear profile considered in this paper. In addition, the other autocorrelation structures, such as Moving Average (MA) and Autoregressive Moving Average (ARMA), could be a fruitful area for future research.

\section{Acknowledgement}

The authors are thankful for precious comments by the respectful anonymous referees which led to significant improvement in the paper.

\section{References}

1. Soleimani, P., Noorossana, R. and Amiri, A. "Simple linear profiles monitoring in the presence of within profile autocorrelation", Computers and Industrial Engineering, 57(3), pp. 1015-1021 (2009).
2. Koosha, M. and Amiri, A. "Generalized linear mixed model for monitoring autocorrelated logistic regression profiles", The International Journal of Advanced Manufacturing Technology, 64(1-4), pp. 487-495 (2013).

3. Jensen, W.A., Birch, J.B. and Woodall, W.H. "Monitoring correlation within linear profiles using mixed models", Journal of Quality Technology, 40(2), pp. 167-183 (2008).

4. Noorossana, R., Amiri, A. and Soleimani, P. "On the monitoring of autocorrelated linear profiles", Communications in Statistics-Theory and Methods, 37(3), pp. 425-442 (2008).

5. Kazemzadeh, R.B., Noorossana, R. and Amiri, A. "Phase II monitoring of autocorrelated polynomial profiles in AR (1) processes", Scientia Iranica, 17(1), pp. 12-24 (2010).

6. Noorossana, R., Saghaei, A. and Dorri, M. "Linear profile monitoring in the presence of non-normali and autocorrelation", International Journal of Industrial Engineering and Production Research, 21(4), pp. 221230 (2010).

7. Soleimani, P., Noorossana, R. and Niaki, S.T.A. "Monitoring autocorrelated multivariate simple linear profiles", The International Journal of Advanced Manufacturing Technology, 67(5-8), pp. 1857-1865 (2013).

8. Narvand, A., Soleimani, P. and Raissi, S. "Phase II monitoring of auto-correlated linear profiles using linear mixed model", Journal of Industrial Engineering International, 9(1), pp. 1-9 (2013).

9. Zhang, Y., He, Z., Zhang, C. and Woodall, W.H. "Control charts for monitoring linear profiles with within-profile correlation using gaussian process models", Quality and Reliability Engineering International, 30(4), pp. 487-501 (2014).

10. Amiri, A. and Allahyari, S. "Change point estimation methods for control chart postsignal diagnostics: a literature review", Quality and Reliability Engineering International, 28(7), pp. 673-685 (2011).

11. Nedumaran, G., Pignatiello Jr, J.J. and Calvin, J.A. "Dentifying the time of a step-change with $\mathrm{x} 2$ control charts", Quality Engineering, 13(2), pp. 153-159 (2000).

12. Sullivan, J.H. "Detection of multiple change points from clustering individual observations", Journal of Quality Technology, 34(4), pp. 371-383 (2002).

13. Perry, M.B., Pignatiello, J.J., Jr. and Simpson, J.R. "Change point estimation for monotonically changing Poisson rates in SPC", International Journal of Production Research, 45(8), pp. 1791-1813 (2007).

14. Perry, M.B. and Pignatiello, J.J., Jr. "Identifying the time of step change in the mean of autocorrelated processes", Journal of Applied Statistics, 37(1), pp. 119-136 (2010).

15. Ghazanfari, M., Alaeddini, A., Akhavan Niaki, S.T. and Aryanezhad, M.B. "A clustering approach to identify the time of a step change in Shewhart control 
charts", Quality and Reliability Engineering International, 24(7), pp. 765-778 (2008).

16. Noorossana, R. and Shadman, A. "Estimating the change point of a normal process mean with a monotonic change", Quality and Reliability Engineering International, 25(1), pp. 79-90 (2009).

17. Torkamani, E.A., Niaki, S.T.A., Aminnayeri, M. and Davoodi, M. "Estimating the change point of correlated poisson count processes", Quality Engineering, 26(2), pp. 182-195 (2014).

18. Mahmoud, M.A., Parker, P.A., Woodall, W.H. and Hawkins, D.M. "A change point method for linear profile data", Quality and Reliability Engineering International, 23(2), pp. 247-268 (2007).

19. Sharafi, A., Aminnayeri, M., Amiri, A. and Rasouli, M. "Estimating the change point of binary profiles with a linear trend disturbance", International Journal of Industrial Engineering, 24(2), pp. 123-129 (2013).

20. Zand, A., Yazdanshenas, N. and Amiri, A. "Change point estimation in phase I monitoring of logistic regression profile", The International Journal of Advanced Manufacturing Technology, 67(9), pp. 23012311 (2013).

21. Sharafi, A., Aminnayeri, M. and Amiri, A. "An MLE approach for estimating the time of step changes in Poisson regression profiles", Scientia Iranica, 20(3), pp. 855-860 (2013).

22. Kazemzadeh, R.B., Noorossana, R. and Ayoubi, M. "Change point estimation of multivariate linear profiles under linear drift", Communications in StatisticsSimulation and Computation, 44(6), pp. 1570-1599 (2015).

23. Keramatpour, M., Niaki, S.T.A., Soleymanian, M.E. and Khedmati, M. "Monitoring and change point estimation of AR (1) autocorrelated polynomial profiles", International Journal of Engineering-Transactions $C$ : Aspects, 26(9), pp. 933-942 (2013).

24. Mirbeik, H., Kazemzadeh, R.B. and Amiri, A. "Step change point estimation in monitoring of autocorrelated simple linear profiles using clustering and maximum likelihood methods", Journal of Industrial Engineering Research in Production Systems, 4(7), pp. 37-47 (2016).

25. Kang, L. and Albin, S. "On-line monitoring when the process yields a linear", Journal of Quality Technology, 32(4), pp. 418-426 (2000).

26. Timmer, D.H., Pignatiello, J.J. and Longnecker, M.T. "The development and evaluation of cusum-based control charts for an AR(1) process", IIE Transactions, 30(3), pp. 525-534 (1998).

\section{Appendix A: Calculations of obtaining the maximum likelihood estimator for the transformed observations}

Initially, logarithm of the likelihood function is written as:

$$
\begin{aligned}
& L n\left[L\left(A_{1}^{\prime \prime}, A_{0}^{\prime \prime}, \tau \mid y^{\prime}\right)\right]=L n\left(\frac{1}{\prod_{j=1}^{T} \prod_{i=1}^{n} \sigma \sqrt{2 \pi}}\right) \\
& -\frac{1}{2 \sigma^{2}}\left(\sum_{j=1}^{\tau} \sum_{i=1}^{n}\left(y_{i j}^{\prime}-\left(A_{0}^{\prime}+A_{1}^{\prime} x_{i}^{\prime}\right)\right)^{2}\right. \\
& \left.+\sum_{j=\tau+1}^{T} \sum_{i=1}^{n}\left(y_{i j}^{\prime}-\left(A_{0}^{\prime \prime}(1-\rho)+A_{1}^{\prime \prime} x_{i}^{\prime}\right)\right)^{2}\right) .
\end{aligned}
$$

Then, by deriving with respect to the parameters of $A_{0}^{\prime \prime}$ and $A_{1}^{\prime \prime}$, a system of equations is obtained as:

$$
\begin{aligned}
& \sum_{j=\tau+1}^{T} \sum_{i=1}^{n} y_{i j}^{\prime}=\sum_{j=\tau+1}^{T} \sum_{i=1}^{n} A_{0}^{\prime \prime}(1-\rho)+\sum_{j=\tau+1}^{T} \sum_{i=1}^{n} A_{1}^{\prime \prime} x_{i}^{\prime}, \\
& \sum_{j=\tau+1}^{T} \sum_{i=1}^{n} x_{i}^{\prime} y_{i j}^{\prime}=\sum_{j=\tau+1}^{T} \sum_{i=1}^{n} A_{0}^{\prime \prime}(1-\rho) x_{i}^{\prime}+\sum_{j=\tau+1}^{T} \sum_{i=1}^{n} A_{1}^{\prime \prime} x_{i}^{\prime 2} .
\end{aligned}
$$

By solving the system of the above equations, we will have:

$$
\begin{aligned}
\hat{A}_{0}^{\prime \prime}= & \left\{\left(\sum_{i=1}^{n} x_{i}^{\prime 2}\right)\left(\sum_{j=\tau+1}^{T} \sum_{i=1}^{n} y_{i j}^{\prime}\right)\right. \\
& \left.-\left(\sum_{i=1}^{n} x_{i}^{\prime}\right)\left(\sum_{j=\tau+1}^{T} \sum_{i=1}^{n} y_{i j}^{\prime} x_{i}^{\prime}\right)\right\} \\
& \div(1-\rho)(T-\tau)\left(n \sum_{i=1}^{n} x_{i}^{\prime 2}-\left(\sum_{i=1}^{n} x_{i}^{\prime}\right)^{2}\right), \\
\hat{A}_{1}^{=}\{ & \left.\left(\sum_{j=\tau+1}^{T} \sum_{i=1}^{n} y_{i j}^{\prime}\right)-n \hat{A}_{0}^{\prime \prime}(1-\rho)(T-\tau)\right\} \\
& \div(T-\tau)\left(\sum_{i=1}^{n} x_{i}^{\prime}\right) .
\end{aligned}
$$

Now, the obtained values for $A_{1}^{\prime \prime}$ and $A_{0}^{\prime \prime}$ are replaced in Eq. (A.1) and by simplifying it, Eq. (A.5) is obtained. Finally, the change point is obtained using Eq. (A.6):

$$
\begin{aligned}
\operatorname{Ln}[ & \left.L\left(A_{1}^{\prime \prime}, A_{0}^{\prime \prime}, \tau \mid y^{\prime}\right)\right] \\
& =-\frac{1}{2 \sigma^{2}}\left\{\sum_{j=\tau+1}^{T} \sum_{i=1}^{n}\left(y_{i j}^{\prime}-\left(\hat{A}_{0}^{\prime \prime}(1-\rho)+\hat{A}_{1}^{\prime \prime} x_{i}^{\prime}\right)\right)^{2}\right. \\
& \left.-\sum_{j=\tau+1}^{T} \sum_{i=1}^{n}\left(y_{i j}^{\prime}-\left(A_{0}^{\prime}+A_{1}^{\prime} x_{i}^{\prime}\right)\right)^{2}\right\}
\end{aligned}
$$




$$
\begin{aligned}
\hat{\tau}=\underset{0 \leq \tau<T}{\arg \max }\left[-\frac{1}{2 \sigma^{2}}\left(\sum _ { j = \tau + 1 } ^ { T } \sum _ { i = 1 } ^ { n } \left(\left(y_{i j}^{\prime}\right.\right.\right.\right. \\
\left.-\left(\hat{A}_{0}^{\prime \prime}(1-\rho)+\hat{A}_{1}^{\prime \prime} x_{i}^{\prime}\right)\right)^{2} \\
\left.-\sum_{j=\tau+1}^{T} \sum_{i=1}^{n}\left(y_{i j}^{\prime}-\left(A_{0}^{\prime}+A_{1}^{\prime} x_{i}^{\prime}\right)\right)^{2}\right] .
\end{aligned}
$$

\section{Appendix B: Computations of the proposed change point estimator}

To obtain the proposed change point estimator in Eq. (28), we first take the partial derivations from the logarithm of the likelihood function given in Eq. (27) with respect to the unknown parameters $A_{0}^{\prime \prime}$ and $A_{1}^{\prime \prime}$, and two equations are obtained as follows:

$$
\begin{aligned}
& \sum_{j=\tau+1}^{T}\left\{-2\left(1-\rho^{2}\right)\left(y_{1 j}-\left(A_{0}^{\prime}+A_{1}^{\prime} x_{1}\right)\right)\right. \\
&+\sum_{i=2}^{n} 2(\rho-1)\left(y_{i j}-\left[\left(A_{0}^{\prime}+A_{1}^{\prime} x_{i}\right)\right.\right. \\
&\left.\left.\left.-\rho\left(A_{0}^{\prime}+A_{1}^{\prime} x_{i-1}\right)\right]-\rho y_{i-1 j}\right)\right\}, \\
& \sum_{j=\tau+1}^{T}\left\{-2 x_{1}\left(1-\rho^{2}\right)\left(y_{1 j}-\left(A_{0}^{\prime}+A_{1}^{\prime} x_{1}\right)\right)\right. \\
&+\sum_{i=2}^{n} 2\left(\rho x_{i-1}-x_{i}\right)\left(y_{i j}-\left[\left(A_{0}^{\prime}+A_{1}^{\prime} x_{i}\right)\right.\right. \\
&\left.\left.\left.-\rho\left(A_{0}^{\prime}+A_{1}^{\prime} x_{i-1}\right)\right]-\rho y_{i-1 j}\right)\right\} .
\end{aligned}
$$

The above equations are put equal to zero, and a system of two equations and two unknowns is created. Then, by solving the equation system, we will have:

$$
\hat{A}_{1}^{\prime}=\frac{p}{c}
$$

where:

$$
\begin{aligned}
p= & \sum_{j=\tau+1}^{T} 2 k\left(1-\rho^{2}\right) y_{i j}-\sum_{j=\tau+1}^{T} \sum_{i=2}^{n} 2 k(\rho-1) y_{i j} \\
& +\sum_{j=\tau+1}^{T} \sum_{i=2}^{n} 2 k \rho(\rho-1) y_{i-1 j} \\
& -\sum_{j=\tau+1}^{T} 2 H x_{1}\left(1-\rho^{2}\right) x_{1}
\end{aligned}
$$

$$
\begin{aligned}
& +\sum_{j=\tau+1}^{T} \sum_{i=2}^{n} 2 H\left(\rho x_{i-1}-x_{i}\right) y_{i j} \\
& -\sum_{j=\tau+1}^{T} \sum_{i=2}^{n} 2 \rho H\left(\rho x_{i-1}-x_{i}\right) y_{i-1 j},
\end{aligned}
$$

and:

$$
\begin{aligned}
c= & -2 k(T-\tau)\left[x_{1}-\sum_{i=2}^{n}\left(\rho x_{i-1}-x_{i}\right)\right] \\
& +2 H(T-\tau)\left[x_{1}^{2}\left(1-\rho^{2}\right)+\left(\rho x_{i-1}-x_{i}\right)^{2}\right] .
\end{aligned}
$$

In the above equations, $k$ and $H$ are defined as Eqs. (B.6) and (B.7), respectively.

$$
\begin{aligned}
& k=2(T-\tau)\left[\left(1-\rho^{2}\right) x_{1}+(\rho-1) \sum_{i=2}^{n}\left(\rho x_{i-1}-x_{i}\right)\right] \\
& H=2(T-\tau)\left[\left(1-\rho^{2}\right)+(n-1)(1-\rho)^{2} !\right] .
\end{aligned}
$$

Also, we have:

$$
\begin{aligned}
\hat{A}_{0}^{\prime}= & \frac{F}{k}, \\
F= & 2 x_{1}\left(1-\rho^{2}\right) \sum_{j=\tau+1}^{T}\left(y_{1 j}-\hat{A}_{1}^{\prime} x_{1}\right) \\
& -2 \sum_{j=\tau+1}^{T} \sum_{i=2}^{n}\left(\rho x_{i-1}-x_{i}\right)\left[y_{i j}\right. \\
& \left.+\hat{A}_{1}^{\prime}\left(\rho x_{i-1}-x_{i}\right)-\rho y_{i-1 j}\right] .
\end{aligned}
$$

$F$ is computed based on Eq. (B.9).

By replacing the estimates of parameters $A_{0}^{\prime}$ and $A_{1}^{\prime}$ in Eq. (B.10), the proposed change point estimator is obtained as Eq. (B.11):

$$
\begin{gathered}
\log \left(L\left(A_{0}, A_{1}, \tau \mid y\right)\right)=\sum_{j=1}^{\tau} \log \left(\left(2 \pi \sigma^{2}\right)^{-n / 2}\left(1-\rho^{2}\right)^{1 / 2}\right) \\
+\sum_{j=1}^{\tau}\left[-\frac{1}{2 \sigma^{2}}\left\{\left(1-\rho^{2}\right)\left(y_{1 j}-\left(A_{0}+A_{1} x_{1}\right)\right)^{2}\right.\right. \\
\left.\left.+\sum_{i=2}^{n}\left(y_{i j}-\delta_{i}-\rho y(i-1) j\right)^{2}\right\}\right] \\
+\sum_{j=\tau+1}^{T} \log \left(\left(2 \pi \sigma^{2}\right)^{-n / 2}\left(1-\rho^{2}\right)^{1 / 2}\right)
\end{gathered}
$$




$$
\begin{aligned}
& +\sum_{j=\tau+1}^{T}\left[-\frac{1}{2 \sigma^{2}}\left\{\left(1-\rho^{2}\right)\left(y_{1 j}-\left(A_{0}^{+} A_{1}^{x_{1}}\right)\right)^{2}\right.\right. \\
& \left.\left.+\sum_{i=2}^{n}\left(y_{i j}-\delta_{i}^{\prime}-\rho y_{(i-1) j}\right)^{2}\right\}\right]
\end{aligned}
$$

$\hat{\tau}=\underset{0 \leq \tau<T}{\arg \max }$

$$
\begin{aligned}
& \left\{\sum _ { j = \tau + 1 } ^ { T } \left[-\frac{1}{2 \sigma^{2}}\left\{\left(1-\rho^{2}\right)\left(y_{1 j}-\left(\hat{A}_{0}^{\prime}+\hat{A}_{1}^{\prime} x_{1}\right)\right)^{2}\right.\right.\right. \\
& \left.\left.+\sum_{i=2}^{n}\left(y_{i j}-\hat{\delta}_{i}^{\prime}-\rho y_{(i-1) j}\right)^{2}\right\}\right] \\
& -\sum_{j=\tau+1}^{T}\left[-\frac{1}{2 \sigma^{2}}\left\{\left(1-\rho^{2}\right)\left(y_{1 j}-\left(A_{0}+A_{1} x_{1}\right)\right)^{2}\right.\right. \\
& \left.\left.\left.+\sum_{i=2}^{n}\left(y_{i j}-\delta_{i}-\rho y_{(i-1) j}\right)^{2}\right\}\right]\right\} .
\end{aligned}
$$

\section{Biographies}

Reza Baradaran Kazemzadeh received a BS degree in Operations Research, an MS degree in Industrial Engineering, and a $\mathrm{PhD}$ degree in Technology Management from the Bradford University, UK. He is currently an Associate Professor in Tarbiat Modares University, Tehran, Iran. He is a member of the Iranian Statistical Association. His research interests include quality en- gineering and management, profile monitoring, applied multivariate statistics, and operations management. His publications have appeared in Quality \& Quantity, the European Journal of Operational Research, Communications in Statistics, the International Journal of Advanced Manufacturing Technology, and Quality and Reliability Engineering International.

Amirhossein Amiri is a Professor in Shahed University in Iran. He holds the BS, MS, and PhD degrees in Industrial Engineering from Khajeh Nasir University of Technology, Iran University of Science and Technology, and Tarbiat Modares University in Iran, respectively. He is now the Vice Chancellor of Education in Faculty of Engineering in Shahed University in Iran and a member of the Iranian Statistical Association. His research interests are statistical quality control, profile monitoring, and Six Sigma. He has published many papers in the area of statistical process control in high quality international journals such as Quality and Reliability Engineering International, Communications in Statistics, Computers and Industrial Engineering, Journal of Statistical Computation and Simulation, Soft Computing, and so on. He has also published a book with John Wiley and Sons in 2011 entitled Statistical Analysis of Profile Monitoring.

Hamidreza Mirbeik holds BS and MS degrees in Industrial Engineering from Azad University, Khoramabad, Iran, and Tarbiat Modares University, Tehran, Iran, respectively. His research interests are time series analysis, profile monitoring, and change point estimation. 